\title{
A Comparison of Current and Emerging Ion and Laser Beam Techniques for High Throughput Material Removal
}

\author{
Srinivas Subramaniam ${ }^{1}$, Kaushik Muthur ${ }^{1}$, K Johnson $^{1}$ and Mclean P. Echlin ${ }^{2}$ \\ 1. Intel Corporation, Chandler, AZ, U.S.A. \\ 2. Materials Department, University of California at Santa Barbara, Santa Barbara, CA, U.S.A.
}

Advances in technology have been instrumental in providing materials engineers and scientists with a broad array of techniques and capabilities to support research and applications driving technology. Beam based techniques have unique advantages in these areas providing the means to use a focused probe to inspect and image regions of interest in an as-is manner as well as progressively by sputtering/ablating the sample to expose new surfaces $[1,2]$. This provides the analyst with the freedom to choose the best technique suited to his/her application. This paper will provide a comparison of some current and emerging techniques and discuss them comparing their advantages and limitations with the aim of understanding the best ways of using these technologies to maximum benefit.

Generic Intel microprocessor samples were used to perform the experiments. The samples were initially ground to remove the package substrates leaving the silicon and interconnect stack intact. Standard focused ion beam (FIB) instrumentation with Gallium and Xenon ion columns were used for milling comparisons on the FIB tools. Laser beam evaluations were performed using a TriBeam tool comprising a modified FIB tool incorporating a femtosecond laser. Argon Ion milling was performed using a broad beam tools. For FIB milling evaluations conventional mounting and coating procedures were used prior to introducing the samples in the respective tools. In the case of the TriBeam, and Argon broad beam tools the samples were cleaved and laser milled close to the region of interest following which they were introduced into the tools for milling and ablation at the cleaved edge.

Figures 1 and 2 shows a representative images of a FIB cross-sections performed using the Xe FIB and Ga FIB respectively. Milling times on the Xe FIB and Ga FIB were $1 \mathrm{hr}$ and 8hrs respectively. The presentation will go into more detailed comparisons and discussions of these techniques while highlighting some of their advantages and limitations [3].

\section{References:}

[1] Subramaniam et al, Microscopy and Microanalysis (2014) p. 296.

[2] Echlin, M.P., et al, Materials Characterization 100 (2015) p. 1.

[3] The authors would like to acknowledge Deepak Goyal for help with reviewing the paper. 
Figure 1. SE image of final surface on sample milled using the Xe FIB

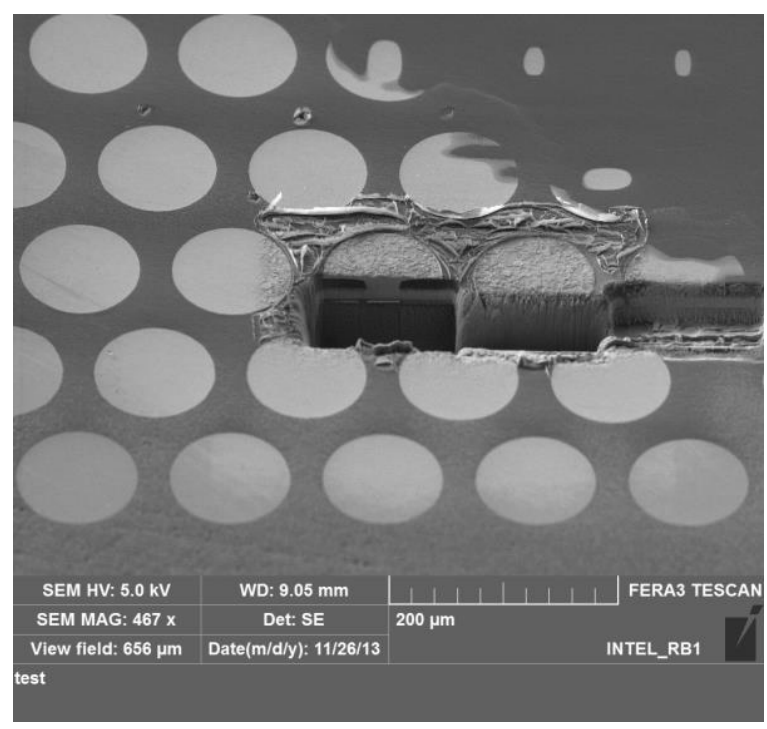

Figure 2. SE image of final surface on sample milled using the Ga FIB

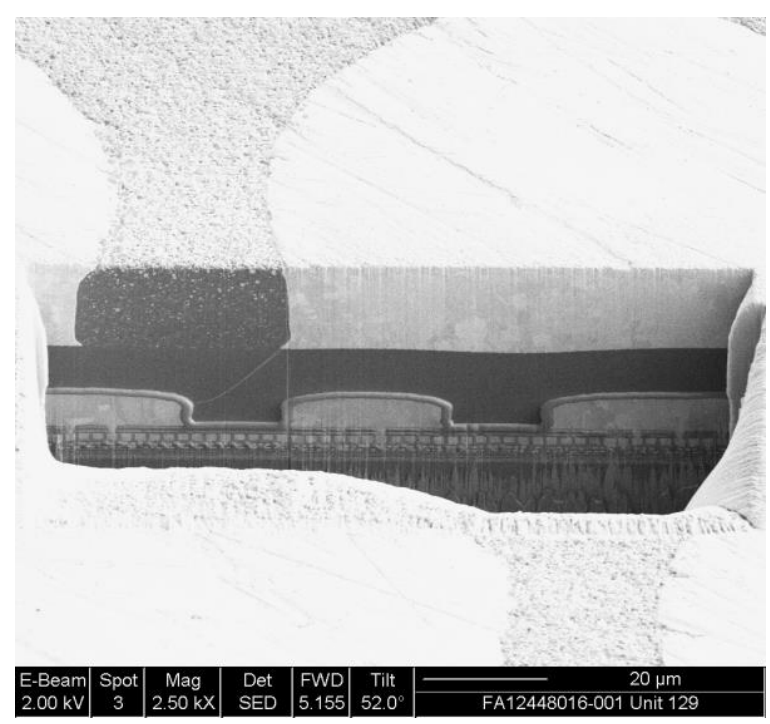

